\title{
The Incidence, Gender Predilection and Etiology of Sacrococcygeal Pilonidal Sinus
}

\author{
Ahmed Naji Abdalrezaq \\ Department of Surgery \\ Shar Teaching Hospital \\ Sulaimani, Iraq \\ Ahmedabdalrezaq82@gmail.com \\ Fadhil Ahmed Mohialdeen \\ Community Health Department \\ Technical College of Health \\ Sulaimani Polytechnic University \\ Sulaimani, Iraq \\ fadhilahmed@hotmail.com
}

\author{
Hawar Hassan Naqshbandi \\ Department of Surgery \\ Shar Teaching Hospital \\ Sulaimani, Iraq \\ hawar.ali@univsul.edu.iq \\ Mohammed IM Gubari \\ Community Health Department \\ Technical College of Health \\ Sulaimani Polytechnic University \\ Sulaimani, Iraq \\ Mohammed.jubari@gmail.com
}

Volume 4 - Special Issue: 3rd International Conference on Health \& Medical Sciences: Insight into Advanced Medical Research (ICHMS 2019)

\section{DOI:}

10.24017/science.2019

.ICHMS.7

Received:

29 May 2019

Accepted:

2 July 2019

\section{Abstract}

Pilonidal Sinus (PNS) is widely distributed in both the emergency department and General Surgery outpatient clinic. Most authors consider the disease as an acquired disease, occurs due to the insertion of broken hairs into the skin with the subsequent tissue inflammation and fibrosis. The incidence of PNS is particularly high in younger males, especially from the Mediterranean region. The aim of the current study was to determine the incidence of the PNS Disease in the city of Sulaimaniyah and the reasons behind the development of the disease. A prospective cohort study was performed in the period between January 2018 and January 2019(1 year) at the Department of Surgery of Shar Teaching Hospital, Sulaimaniyah City. All consecutive patients who presented to the Emergency Department or outpatient clinic and admitted to the Shar Hospital were investigated for the presence of Sacrococcygeal Pilonidal Sinus Disease. Those who found to have the disease were offered surgical excision and examination of the excised specimen for evaluation of the etiology of the Disease. Overall, 103 out of 1000 patients had Pilonidal sinus, while 897 out of 1000 patients did not show any sign of the disease. Fifty-three out of 103 patients with Pilonidal Sinus were symptomatic and the rest were asymptomatic. The factors which associated with the presence of PNS were: family history (23/103 compared with 45/897, $<<0.0001$ ); overweight defined as BMI > 25 (39/103 compared with 213/897, $p<0.0001)$; having excessive body hair distribution (41/103 compared with 122/897, $p<0.05)$. $P N S$ is considered one of the acquired clinical conditions, that is occurred due to insertion of a hair shaft into the skin, followed by subsequent local inflammation and granulation tissue formation. 


\section{If the etiology is clearly identified, the condition can be readily prevented.}

Keywords: Sacrococcygeal, Pilonidal Sinus, Sulaimaniyah City, hair shaft insertion, natal cleft, adult, male

\section{INTRODUCTION}

Herbert Mayo was one of the early scientists who was first described the PNS as a sinus containing hair follicle in the Sacrococcygeal area in a woman, in 1880. Hodges is one of the scientists who used the term "Pilonidal" which that derived from Latin 'pilus' means hair and 'nidus' which means nest, in 1883[1]. PNS is defined as an acute or chronic infectious process in the subcutaneous tissue, leading to the formation of a sinus tract lined by epithelial tissue. While Pilonidal Sinus may occur at different areas across skin folds, this includes interdigital web spaces, umbilicus, and genital area; natal (intergluteal) cleft is reported as the most common site for the development of the nidal Sinus Disease [1-4]. PNS is a common clinical condition with an estimated incidence is 26 / 100, 000 and a male / female ratio of 3-4:1[2]. Certain areas around the world show a higher percentage of the disease in comparison to others, particularly in the Mediterranean, therefore many studies were trying to search for the reasons behind this contrast. While some authors attribute these higher percentages to hormonal changes, others arguing that such geographical difference could be related to local factors and personal behavior and attitude $[5,6]$. This theory is backstopped by the fact that Pilonidal sinuses are mainly found in the peripubertal period where the hormonal changes are peaked. Despite the fact that PNS can be asymptomatic, most patients seek medical care when the sinus gets infected and becomes an abscess [7]. Accurate and prompt diagnosis of Pilonidal Sinus is not difficult; however, it is the increased rate of failure of management and the high recurrence rate after initial treatment made the PNS Disease one of the most frustrating diseases for both the patient and the surgeon [8]. Knowledge of the etiology of the disease is essential for the management; most sinuses develop due to hair shaft insertion into the skin associated with repeated local trauma, leading to local inflammatory reaction ending with chronic sinus formation [9]. As a result, the heated controversy associated with the etiology of Pilonidal disease, whether congenital or acquired, has led to a wide array of treatments modalities with different outcomes. Many procedures were invented for the management of Pilonidal Sinus including surgical such as incision and drainage which increasingly applied in the management of the disease on an emergency base [10]. Other procedures like de-roofing and excision with the wound left to heal by secondary intention are widely used nowadays with significantly acceptable recurrence rates [11]. Newer modalities of management like excision and flaps cover, Karydakis procedure and MacFee technique are getting more popularity $[12,13]$. The choice among these arrays of options largely depends on surgeon's familiarity with the proposed procedure, the amount of time needed for the residual cavity to heal and the presumptive lower recurrence rates of a certain procedure [14]. The least recurrence rates were associated with sinus excision with primary closure using a lateral approach. As a result of the high recurrence rate that is usually associate surgical approaches, a couple of conservative remedies have been described to show some acceptable results. Phenol solution and alcohol injection in the sinus tract were shown to be able to mitigate the symptoms of the disease. Many other studies mentioned more favorable outcomes when a combination of medical and surgical interventions was used; however, no consensus was obtained [25]. This study is designed to search for the possible conditions and risk factors that are contributing to the development of the sinus disease. In addition to that, the incidence and gender distribution are tried to be addressed in Sulaimaniyah City, Iraq. 


\section{METHODS AND MATERIALS}

The current investigation was carried out over a one-year period, starting from January 2018 until January 2019. The study sample included 1000 patients who are citizens of the city of Sulaimaniyah, Iraq. A local ethical committee approved a questionnaire that was prepared; answers for certain questions were obtained from those who participated in the study. These questions included the age and gender of the participant, occupation, and family history of sacral Sinus disease or elsewhere in the body. The personal hygiene, in terms of frequency of bathing, was also assessed during the candidate interview. The time spent in a vehicle was one of the factors that used to be considered as a risk factor that leads to PNS disease. This factor has been taken into consideration whether this time was spent as a driver of a vehicle or as a passenger. For the sake of simplifying the evaluation, some answers were divided into two categories: ("Yes" and "No" or "1" and "0"), for example, the frequency of bathing was recorded as "1" if it was more than once daily and " 0 " if it was less. Preceding the questionnaire, a thorough physical examination was performed, in which the patients' weight and height were recorded and their body mass index was calculated. The presence of excessive hair over the body and skin appearance (dark skin or fair skin) was also noted. Finally, the patients were examined for the presence or absence of sacrococcygeal Pilonidal sinus. For those who tested positive for the disease, surgical excision was the preferred method of management in this study in order to sample the disease and study the microscopical features of the sampled tissues. Afterward, the collected data were administered automatically into a database and the Statistical Package for the Social Sciences (SPSS) program has been used for statistical analysis. The collected data were categorized into two main groups: those who were having Pilonidal sinus $(n=103)$ and those who had not $(n=897)$. P-value of less than 0.05 was considered as significant. Patients with positive findings, such as chronic discharging sinus or abscess and asymptomatic patients as well, have been offered a surgical excision at the Shar Teaching Hospital and a Histopathological examination of their resected diseases. Eventually, only twenty-nine out of the one hundred and nine patients affected by the disease could be persuaded to have their condition surgically treated under our care.

\section{RESULTS}

A total of 1000 patients were enrolled in the current study and were randomly selected from different age groups. The group of 20-29 years had the highest percentage of patients who were suffering from the condition. The results are presented summarized in (table 1). Furthermore, this study showed that both males and females were liable to the Pilonidal disease with a gender male/ female ratio of 2.4:1. As patients were screened for the possible causes of Pilonidal Sinus and risk factors that might contribute to this condition, familial history of Pilonidal Sinus was a significant finding in this study with a p-value of $<0.0001$ (Table 2). A 103 of candidates were found to have Sacrococcygeal Pilonidal Sinus. Fifty-six patients out of the one hundred and three patients were presented with different complaints. Symptoms include, painful swelling, abscess formation, and chronic discharging sinus were recorded among this group. The other 47 , on the other hand, were asymptomatic, only to be discovered to have the condition after physical examination with a well-formed sinus tract in the sacrococcygeal area.

Table 1 Gender and age distribution of the patients

\begin{tabular}{|l|l|l|}
\hline Factors investigated & Pilonidal sinus $(\mathbf{n}=\mathbf{1 0 3})$ & No Pilonidal sinus (n=897) \\
\hline Gender & & \\
\hline Male & $73(70.8)$ & $523(58.3)$ \\
\hline Female & $30(29.1)$ & $374(41.6)$ \\
\hline Age groups & & \\
\hline $10-19$ years & 35 & 33.9 \\
\hline $20-29$ years & 51 & 49.5 \\
\hline $30-39$ years & 13 & 12.6 \\
\hline$>40$ years & 4 & 3.8 \\
\hline
\end{tabular}


Table 2 Number (percentage) of patients investigated for the cause of Pilonidal sinus

\begin{tabular}{|l|c|c|}
\hline \multicolumn{1}{|c|}{ Factors investigated } & $\begin{array}{c}\text { Pilonidal sinus } \\
(\mathbf{n = 1 0 3 )}\end{array}$ & $\begin{array}{c}\text { No Pilonidal sinus } \\
(\mathbf{n}=\mathbf{8 9 7})\end{array}$ \\
\hline Family history & $45(5.01)^{*}$ & $23(22.3)$ \\
\hline $\begin{array}{l}\text { Personal hygiene ( Bathing more frequent } \\
\text { than every other day }\end{array}$ & $266(29.6)$ & $22(21.3)$ \\
\hline $\begin{array}{l}\text { Time spent in a vehicle (at least } \\
\text { 100km/week) }\end{array}$ & $320(35.6)$ & $41(39.8)$ \\
\hline 25)>Body weight (BMI & $213(23.7)^{*}$ & $39(37.8)$ \\
\hline Excessive Hair & $122(13.6) *$ & $41(39.8)$ \\
\hline Skin appearance (dark skinned) & $722(80.4)$ & $79(76.6)$ \\
\hline
\end{tabular}

* $\mathrm{P}$ value $<0.05$

Results also revealed that patients with a BMI of $>25$ are more liable for developing Pilonidal Sinus in compare to those with normal BMI (Table 2). Likewise, abundant body hair status was noticed to be significant in patients who were affected by the disease with a p-value of $<0.05$.

Two more criteria that are known to be associated with an increased incidence for the development of PNS were investigated in this study, namely the personal hygiene and the amount of time spent sitting in a moving vehicle. Those two figures were addressed in terms of frequency of bathing and the number of hours spent in a vehicle per week, respectively. However, results showed both two factors had only minor significance to be considered as leading causes to acquire the disease (Tables $3 \& 4$ )

Table 3 Association between the times spent driving a vehicle and formation of Pilonidal sinus

\begin{tabular}{|l|l|l|}
\hline At least 100km|week & Pilonidal sinus $\mathrm{n}=103$ & No Pilonidal sinus $\mathrm{n}=897$ \\
\hline Yes & $41(39.8)$ & $320(35.6)$ \\
\hline No & $62(60.1)$ & $577(64.3)$ \\
\hline
\end{tabular}

P-value 0.4485

Table 4 Effect of bathing frequency on the formation of Pilonidal sinus

\begin{tabular}{|l|l|l|}
\hline Frequency of bathing & Pilonidal sinus $\mathrm{n}=103$ & No Pilonidal sinus $\mathrm{n}=897$ \\
\hline More than every other day & $22(21.3)$ & $266(29.6)$ \\
\hline Less than every other day & $81(78.6)$ & $631(70.3)$ \\
\hline
\end{tabular}

P-value 0.0851

Finally, our study investigated the skin appearance as a possible contributor to developing the disease, however, results showed an insignificant relation.

\section{DISCUSSION}

PNS disease is a common condition among young people, being highest in those aged between 15 and 30 years old [26]. Such high presentation of the disease at this particular age group with a mean of 22 years, draw the attention to the hypothesis that relates this figure to the effect of hormonal changes during puberty and the subsequent following years. This hormonal imbalance increases the chance of developing boils and folliculitis in the dermal layer of the skin, which ultimately will lead to a local inflammatory reaction that renders the area extremely friable and easily to be penetrated by hair shaft or other local invaders leading eventually to the development of the PNS. A hormonal change which is encountered in the peri-pubertal period is suggested to be the reason $[27,28]$. In this study, male patients were more prone to develop 
Pilonidal Sinus in comparison to females, 73 (70.8\%) of those who had the condition were male patient, while the others were females. Such results with a p-value of less than 0.01 , it was significant enough to consider male gender as a significant risk factor for the development of Pilonidal disease.

Historically, the Sinus Disease was considered as an acquired illness. Hair shaft penetration into the skin plays a major role in the Pathophysiology [29-31]. In our study, 29 out of 103 of patients who had the condition accepted standard surgical excision under our care. After excision, resected specimens were examined under the microscope by the histopathologist who reports that $24(82.7 \%)$ of the excised specimens revealed the presence of hair within the sinus tract. Such results support the theory that the PNS disease is an acquired clinical condition due to broken hair penetration at its end root into subcutaneous tissue and the skin[16].

Loose hair accumulation and penetration into the skin thought to be related to personal hygiene. It is a fact that regular bathing will remove any broken hair or debris from the cleft area. The higher incidence among soldiers in the Second World War drew the attention to such relation. Perhaps due to the less attention to personal hygiene in relation to the battlefield circumstances and limited bathing frequency. In this study, Personal hygiene was considered as a risk factor for the development of the PNS; however, results show an insignificant correlation in personal hygiene in terms of frequent bathing and the development of Pilonidal Sinus.

According to many studies, obesity has a potential contribution to the development of Pilonidal disease $[8,9,11,14]$. It is a fact that obesity increase the depth of the natal cleft and this facilitate the accumulation of hair and other debris, along with concomitant factors such as increased friction and local repeated trauma increasing the tendency of local skin maceration at depth and aiding in the progression of PNS Pathophysiology. We noted that obesity and the ratio of weight to height (body mass index) are factors associated with an increased incidence of Pilonidal Sinus. A 39 (37.8) out of the 103 of those who complained from the disease were overweighted. On the other hand, 684 (76.2) of candidates were not complaining of the disease, were normally weighted during the physical exam. This was significant enough to assume that obesity is an independent risk factor for the disease.

Historically, PNS was considered to be an acquired condition that is caused mainly due to the hair follicles infection and inflammation. Hyperkeratosis plays a major role in the Pathophysiology by obstructing the hair follicle followed by subsequent dilation and rupture of the follicle leading to secondary infection, fistulae and abscess formation[30]. On the contrary of that, some studies hypothesized that fistulae occur only when free hair perforates into an intact skin, and usually are broken hair which has been detached from their follicles in the head, neck or other places from the body [31]. In this study, 39.8\% of patients with Pilonidal Sinus had excessive body hair distribution. This result was significantly enough, p-value 0.016 , to consider excessive hair distribution as an independent risk factor for the development of the Sinus disease.

The positive family history of Pilonidal sinus places an increased incidence of the early development of the condition in a first-degree relative and associated with a higher percentage of the disease recurrence after surgical excision [32]. Such family tendency was the pivotal evidence for the supporters of the theory of congenital background of the disease [10, 12]. Comparatively equal to similar studies about the same topic, our results showed a significant correlation between family history and the development of Pilonidal disease. This correlation could also be attributed to similar hair characteristics, body shape or similar lifestyle.

Sacrococcygeal Sinus disease is known to be more common in those who spend a long time in a moving vehicle. Terms like 'Jeep Disease' or ' Army Disease' were first described in literature during the World War II as a result of the raised number of soldiers and officers with the 
condition especially among those who were the drivers or passengers in the combating armies' vehicles. Some studies suggested this is to be caused by the recurrent local trauma and persistent pressure placed on the buttock and natal cleft area which eventually lead to increase the chance for local skin maceration and hair inoculation into the skin [6]. However, in this study, results suggest no significant correlation between time spent in a vehicle, whether being a passenger or a driver, and the incidence of development of the Disease.

It is acknowledged that Pilonidal sinus occurs more frequently in dark men in comparison to light-colored skin women [33]. Darker-skinned men have more hair on their bodies and this was incriminated to be the culprit behind the increased incidence of the PNS disease among those groups of people. However, our results were insignificant to the skin color and both dark and light skin colored were shown to have equal chances to acquire the disease.

The incidence of PNS is different between races, being more common in Asians and Mediterranean [11, 33]. Many studies suggest different factors were the culprit behind this phenomenon. Of those with interesting relations was the hirsute state of the person. Our study was conducted among the local citizens of the city of Sulaimaniyah, where the vast majority of them are from Kurdish ethnicity. The incidence of the disease in this study was $10.3 \%$. A similar study conducted among 1000 Turkish soldiers showed a similar incidence of 9\% [33].

\section{CONCLUSION}

This study concluded that Pilonidal sinus is an acquired condition that occurs due to the penetration of hair shaft into the skin in vulnerable areas. The microscopic and gross pathologic findings favor the theory of acquired origin over the congenital theory. This was supported by the finding of broken hairs in the majority of the examined resected tissues in the study. This leading us to the conclusion that hair shaft penetration into the skin has an essential role in the development of PNS disease. Such a process, with the adjunct of local tissue trauma and inflammatory process within the affected area, trigger an inflammatory reaction leading, eventually, to the development of the sinus. In regard to the incidence of the disease, we find that PNS was higher among men in contrast to women. Such contrast was mentioned in many other literatures. One of the targets of this study was to calculate the incidence of the disease in the city of Sulaimaniyah. It was found that a substantially high percentage of people who were included in this study were found to be having the disease. We recommend that factors such as hormonal abnormalities and variations to be studied in future studies. In terms of disease prevention, the incidence of the disease can be readily reduced when certain measures are followed. These include, but not limited to, attention to personal hygiene, weight reduction and avoiding improper sitting that put excessive pressure on the natal cleft area. These measures are particularly useful in those with positive family history, as they have a higher risk to develop the disease at an early age than the general population.

\section{REFERENCE}

[1] H. Mayo, "Observations on injuries and diseases of the rectum," The American Journal of the Medical Sciences, vol. 28, p. 435, 1834.

[2] R. Hodges, "Boston med. surg. J., 103. 485. Patey, DH. and Scarfl. RW (1946)," Lancet, vol. 2, p. $484,1880$.

[3] T. L. Hull and J. Wu, "Pilonidal disease," Surgical Clinics, vol. 82, pp. 1169-1185, 2002.

[4] A. K. Ahmed, I. McCallum, P. M. King, and J. Bruce, "Healing by primary versus secondary intention after surgical treatment for pilonidal sinus," Cochrane database of systematic reviews, 2010.

[5] A. Khanna and J. L. Rombeau, "Pilonidal disease," Clinics in colon and rectal surgery, vol. 24, pp. 046053, 2011.

[6] I. Buie, "Jeep disease (pilonidal disease of mechanized warfare)," South Med J, vol. 37, pp. 103-109, 1944.

[7] R. M. HARDAWAY, "Pilonidal cyst—neither pilonidal nor cyst," AMA archives of surgery, vol. 76, pp. 143-147, 1958.

[8] R. W. Dwight and J. K. Maloy, "Pilonidal sinus: experience with 449 cases," New England Journal of Medicine, vol. 249, pp. 926-930, 1953.

[9] J. W. Chamberlain and G. F. Vawter, "The congenital origin of pilonidal sinus," Journal of pediatric surgery, vol. 9, pp. 441-444, 1974. 
[10] G. Karydakis, "New approach to the problem of pilonidal sinus," The Lancet, vol. 302, pp. 1414-1415, 1973.

[11] L. B. Holmes and E. A. Turner, "Hereditary pilonidal sinus," Jama, vol. 209, pp. 1525-1526, 1969.

[12] K. Søndenaa and M. Pollard, "Histology of chronic pilonidal sinus," Apmis, vol. 103, pp. 267-272, 1995.

[13] G. E. Karydakis, "Easy and successful treatment of pilonidal sinus after explanation of its causative process," Australian and New Zealand Journal of Surgery, vol. 62, pp. 385-389, 1992.

[14] H. P. Kooistra, "Pilonidal sinuses: review of the literature and report of three hundred fifty cases," The American Journal of Surgery, vol. 55, pp. 3-17, 1942.

[15] B. Page, "The entry of hair into a pilonidal sinus," British Journal of Surgery, vol. 56, pp. 32-32, 1969.

[16] F. Stephens and R. Stephens, "Pilonidal sinus: management objectives," Australian and New Zealand Journal of Surgery, vol. 65, pp. 558-560, 1995.

[17] J. Bascom and T. Bascom, "Utility of the cleft lift procedure in refractory pilonidal disease," The American journal of surgery, vol. 193, pp. 606-609, 2007.

[18] B. H. Kanat and S. Sözen, "Disease that should be remembered: Sacrococcygeal pilonidal sinus disease and short history," World Journal of Clinical Cases: WJCC, vol. 3, p. 876, 2015.

[19] B. Maurice and R. Greenwood, "A conservative treatment of pilondidal sinus," British Journal of Surgery, vol. 51, pp. 510-512, 1964.

[20] M. Girgin, B. H. Kanat, R. Ayten, Z. Cetinkaya, Z. Kanat, A. Bozdağ, et al., "Minimally invasive treatment of pilonidal disease: crystallized phenol and laser depilation," International surgery, vol. 97, pp. 288-292, 2013.

[21] M. Girgin and B. H. Kanat, "The results of a one-time crystallized phenol application for pilonidal sinus disease," Indian Journal of Surgery, vol. 76, pp. 17-20, 2014.

[22] A. M. Salih, F. H. Kakamad, R. Q. Salih, S. H. Mohammed, I. J. Habibullah, Z. D. Hammood, et al., "Nonoperative management of pilonidal sinus disease: one more step toward the ideal management therapy-a randomized controlled trial," Surgery, vol. 164, pp. 66-70, 2018.

[23] A. M. Salih, F. H. Kakamad, R. Q. Salih, H. O. Baba, S. H. Mohammed, K. A. Najar, et al., "Pilonidal sinus of neck: A case report," Int J Case Rep Images, vol. 9, p. 100882Z01AS2018, 2018.

[24] S. Emir, Ö. Topuz, B. H. Kanat, and İ. Bali, "Sinotomy technique versus surgical excision with primary closure technique in pilonidal sinus disease," Bosnian journal of basic medical sciences, vol. 14, p. 263, 2014.

[25] B. H. Kanat, M. B. Bozan, F. Mehmet, M. Yur, F. Erol, Z. Özkan, et al., "Comparison Of Early Surgery (Unroofing Curettage) And Elective Surgery (Karydakis Flap Technique) In Pilonidal Sinus Abscess Cases," 2014.

[26] S. Jensen and H. Harling, "Prognosis after simple incision and drainage for a first-episode acute pilonidal abscess," British journal of surgery, vol. 75, pp. 60-61, 1988.

[27] G. Plewig and A. M. Kligman, Acne: morphogenesis and treatment: Springer Science \& Business Media, 2012.

[28] J. Bascom, "Pilonidal disease: origin from follicles of hairs and results of follicle removal as treatment," Surgery, vol. 87, pp. 567-572, 1980.

[29] D. Doll, E. Matevossian, K. Wietelmann, T. Evers, M. Kriner, and S. Petersen, "Family history of pilonidal sinus predisposes to earlier onset of disease and a 50\% long-term recurrence rate," Diseases of the colon \& rectum, vol. 52, pp. 1610-1615, 2009.

[30] F. Bosche, M. M. Luedi, D. van der Zypen, P. Moersdorf, B. Krapohl, and D. Doll, "The hair in the sinus: sharp-ended rootless head hair fragments can be found in large amounts in pilonidal sinus nests," World journal of surgery, vol. 42, pp. 567-573, 2018.

[31] G. A. Chatzoulis, D. Pharmakis, K. Milias, K. Ioannidis, G. Tzikos, D. Delligianidis, et al., "“Jeep Disease” and Optimal Treatment Strategy for Sacrococcygeal Pilonidal Sinus in a High Volume Tertiary Military Medical Center," Hellenic Journal of Surgery, vol. 91, pp. 14-21, 2019.

[32] T. Allen-Mersh, "Pilonidal sinus: finding the right track for treatment," British Journal of Surgery, vol. 77, pp. 123-132, 1990.

[33] Ö. F. Akinci, M. Bozer, A. Uzunköy, Ş. A. Düzgün, and A. Coşkun, "Incidence and aetiological factors in pilonidal sinus among Turkish soldiers," The European journal of surgery, vol. 165, pp. 339-342, 1999. 